\title{
SEGURIDAD ALIMENTARIA EN LOS HOGARES DESDE LA PERSPECTIVA DE UNA POBLACIÓN UNIVERSITARIA
}

Food security in the household from the perspective of a university population

Delia Guillermina González-Aguilar, Zoila Gómez-Cruz, Patricia Landeros-Ramírez, Kenya Regina Morales-Ángel y Carlos Alberto Campos-Bravo*.

Departamento de Salud Pública. Centro Universitario de Ciencias Biológicas y Agropecuarias. Universidad de Guadalajara. Camino Ramón Padilla Sánchez No. 2100 Nextipac, Zapopan, Jalisco. C.P.45200.

*Autor para correspondencia: carlos.cbravo@academicos.udg.mx

\section{Resumen}

La inseguridad alimentaria es un problema de salud pública en todo el mundo, ya que un número creciente de hogares presentan esta situación de vulnerabilidad. El objetivo del presente estudio fue realizar un diagnóstico de la situación respecto a la seguridad alimentaria en los hogares de los estudiantes del Centro Universitario de Ciencias Biológicas y Agropecuarias de la Universidad de Guadalajara, en México. Se aplicó una encuesta en línea a 225 alumnos de licenciatura a través de una plataforma con base en la Escala Latinoamericana y Caribeña de Seguridad Alimentaria. El $52 \%$ de los hogares presenta algún nivel de inseguridad alimentaria, de los cuales 76 presentan
Abstract
Food insecurity is a public health problem throughout the world, since a growing number of households present this situation of vulnerability. The objective of this study was to carry out a diagnosis of the situation regarding food security in

inseguridad leve $(33.77 \%), \quad 30$ inseguridad moderada (13.33 \%) y 11 inseguridad severa (4.88\%). La mayor cantidad de respuestas "sí" (85), fue para la pregunta "¿Alguna vez te preocupó que los alimentos se acabaran en tu hogar?”. Los datos presentados sugieren una realidad preocupante en torno a la seguridad alimentaria en las familias de los estudiantes del Centro Universitario, cinco de cada 10 estudiantes viven en familias con inseguridad alimentaria, es decir, los estudiantes están sufriendo hambre y malnutrición y se muestran preocupados por ello.

Palabras clave: Estudiantes, vulnerabilidad alimentaria, diagnóstico.

the homes of the students of the University Center for Biological and Agricultural Sciences of the University of Guadalajara, in Mexico. An online survey was applied to 225 undergraduate students through a platform based on the Latin American and Caribbean Scale of Food Security. $52 \%$ of the households 
present some level of food insecurity, of which 76 present mild insecurity $(33.77 \%), \quad 30$ moderate insecurity $(13.33 \%)$ and 11 severe insecurity $(4.88 \%)$. The largest number of "yes" answers (85) was for the question "Have you ever worried that food would run out at home?". The data presented suggest a worrying reality regarding food security

\section{Introducción}

En años recientes se han hecho presentes en México diversas problemáticas respecto a los alimentos y la alimentación (excesiva importación de alimentos, abandono del campo, desnutrición y dependencia del exterior para el abasto de alimentos, entre otros). En este contexto, las universidades pueden ser catalizadoras del cambio con un enfoque sistémico, al tomar un papel más activo que impulse el cambio hacia una visión sustentable, de protección de la diversidad biológica y cultural, que considere sistemas de producción y consumo de alimentos que ya están arraigados en el país (EscalonaAguilar, Leal-Ascencio, Pineda-López, Ruíz-Cervantes y Sánchez-Velásquez, 2015).

La Organización de las Naciones Unidas para la Alimentación y la Agricultura

(FAO/FIDA/OS/WFP/UNICEF, 2020), establece que existe seguridad alimentaria y nutricia, cuando los individuos tienen en todo momento acceso físico $\mathrm{y}$ económico a suficientes alimentos inocuos y nutritivos para satisfacer sus necesidades y sus preferencias, a fin de llevar una vida activa y sana.

Los modelos económicos globales, han llevado a tener consecuencias sociales, in the families of the University Center students, five out of 10 students live in families with food insecurity, that is, the students are suffering from hunger and malnutrition and are concerned about it.

Keywords: Students, food vulnerability, diagnosis.

ambientales, culturales y económicas que se reflejan en problemas de inseguridad alimentaria, sobre todo en la población con menor ingreso económico (Altieri, Koohafkan y Giménez, 2012). Es un hecho bien conocido que los cambios a nivel mundial han llevado a cambios regionales y locales, entre los que se pueden mencionar los hábitos de alimentación y el acceso a los mismos. El consumo de alimentos fuera del hogar ha sido uno de los cambios importantes en el estilo de vida en las últimas décadas (Lachat et al., 2012).

La inseguridad alimentaria se considera en años recientes como un importante problema de salud pública en E.U., debido al número de hogares que subsisten bajo esa condición y a su asociación con consecuencias negativas como bajo rendimiento académico, salud deficiente e incluso síntomas como depresión y ansiedad, reflejo de alteraciones en la salud mental (ColemanJensen, Rabbitt, Gregory and Singh, 2016; Goldrick-Rab, Broton, and Eisenberg, 2015; Gundersen and Ziliak 2015; Knol, Robb, McKinley y Wood, 2017). La inseguridad alimentaria es un costo frecuentemente oculto de asistir a la universidad (Broton y Goldrick-Rab, 2018). 
En México los estudios sobre seguridad alimentaria y nutricia a pesar de que se realizan desde diferentes perspectivas (Rosas, Córdova, Villegas y Morales, 2017), aún no abarcan los diferentes aspectos para conocer la situación real en condiciones particulares.

De acuerdo a datos del Consejo Nacional de Evaluación de la Política de Desarrollo Social [CONEVAL], la alimentación es uno de los derechos sociales, sin embargo, en 2018, $20.4 \%$ de la población (25.5 millones de personas), tuvieron carencias por acceso a la alimentación. En ese mismo año, el ingreso económico de 61.1 millones de mexicanos (48.8 \%), no fue suficiente para adquirir la canasta alimentaria y no alimentaria (CONEVAL, 2019), lo cual hace evidente que el ingreso económico es condicionante importante para una alimentación adecuada.

Para el año 2018, en el Estado de Jalisco se reportó un $28.4 \%$ de población en situación de pobreza (2337.6 miles de personas). Jalisco esta ubicado entre los 17 Estados con 10 a $20 \%$ de carencia en alimentación. Entre los 15 municipios o demarcaciones del país, con el mayor número de personas en situación de pobreza en 2015, se encuentran dos pertenecientes a Jalisco: Zapopan $(382,961$ personas) y Guadalajara, (370,890) (CONEVAL, 2019), situación preocupante que va asociada a carencias alimenticias.

La FAO (2019 a,b), recomienda la integración de diversas acciones encaminadas a "Una alimentación sana para un mundo \#HAMBRE CERO”, las cuales enmarca en el involucramiento de los sectores publico, privado y social.
Para lograr lo anterior se requiere de estrategias locales que desde la universidad, coadyuven a la mitigación del problema del hambre, lo cual coincide con las políticas nacionales que están enfocadas en el ámbito de los derechos sociales a la accesibilidad, disponibilidad y calidad, que específicamente en cuanto a la alimentación está orientada a: seguridad alimentaria, adecuación y diversidad de la dieta y combate a la malnutrición, mejorando la accesibilidad económica a los alimentos, así como la calidad de las dietas (CONEVAL, 2019; SS/INSP/INEGI, 2019). Sin embargo, las soluciones a la inseguridad y a la inequidad alimentaria que se han planteado como políticas públicas únicamente están llevando a mayores desequilibrios e inviabilidad (EscalonaAguilar et al., 2015).

En estos tiempos de contingencia en los ámbitos de salud y economía, es prioritario abordar los procesos educativos y de vida de los estudiantes universitarios de una manera integral para que como lo mencionan Escalona-Aguilar et al. (2015), se forme a los futuros profesionales con mentalidad que impacte favorablemente la sustentabilidad socioambiental como base para alcanzar la seguridad y soberanía alimentaria, sin embargo, eso no se logrará satisfactoriamente si durante su trayecto en la universidad se ven sometidos a diversos factores estresantes como la dificultad e incluso imposibilidad en algunos casos para solventar satisfactoriamente su alimentación. La población universitaria es un grupo vulnerable desde el punto de vista nutricional, ya que se caracteriza por saltarse comidas con frecuencia (López et al., 2003). 
El objetivo del presente estudio fue realizar un diagnóstico de la situación en el Centro Universitario de Ciencias Biológicas y Agropecuarias (CUCBA), respecto a la seguridad alimentaria en los hogares de los estudiantes.

\section{Materiales y métodos}

Se aplicó una encuesta en línea a alumnos de licenciatura del CUCBA (Ciencia de los Alimentos [CA], Medicina Veterinaria [MV] y Agronegocios [AN]), a través de la plataforma Google ${ }^{\circledR} \quad$ (sección formularios), que constó de dos apartados: A. Datos generales de identificación; B. Seguridad alimentaria evaluada con base en la Escala Latinoamericana y Caribeña de Seguridad Alimentaria (ELCSA), con ocho preguntas para hogares formados sólo por adultos y 15 preguntas para hogares con adultos y menores de 18 años que fueron evaluadas de acuerdo a la información del Cuadro 1 (FAO, 2012). El tamaño de muestra fue de 225 encuestas. Para el análisis estadístico, se utilizaron tablas de contingencia y prueba de $\mathrm{X}^{2}$ a un nivel de confianza del $95 \%$ (Statgraphics 18 X64, 2019).

Cuadro 1. Puntaje para la clasificación de la seguridad alimentaria según tipo de hogar

\begin{tabular}{lllll}
\hline \multirow{2}{*}{ Tipo de hogar } & \multicolumn{3}{c}{ Clasificación de la seguridad alimentaria } \\
\cline { 2 - 5 } & Seguridad & $\begin{array}{l}\text { Inseguridad } \\
\text { leve }\end{array}$ & $\begin{array}{l}\text { Inseguridad } \\
\text { moderada }\end{array}$ & $\begin{array}{l}\text { Inseguridad } \\
\text { severa }\end{array}$ \\
\hline $\begin{array}{l}\text { Hogares integrados solamente por personas adultas } \\
\text { Hogares integrados por personas adultas y menores } \\
\text { de 18 años }\end{array}$ & 0 & 1 a 3 & 4 a 6 & 7 a 8 \\
\hline
\end{tabular}

\section{Resultados}

De los 225 encuestados, 73 fueron hombres (39 de AN; 24 de CA) y 152 mujeres (127 de CA; 25 de MV). Al analizar los tipos de hogares de acuerdo al nivel de seguridad alimentaria (Seguridad/Inseguridad) (Cuadro 2), se encontró diferencia significativa $(\mathrm{p}<0.82)$. El $52 \%$ de los hogares presenta algún nivel de inseguridad alimentaria.

Cuadro 2. Evaluación de la seguridad alimentaria según tipo de hogar de los alumnos del CUCBA

\begin{tabular}{|c|c|c|c|c|c|c|}
\hline \multirow{3}{*}{$\begin{array}{l}\text { Clasificación de la } \\
\text { seguridad alimentaria }\end{array}$} & \multicolumn{4}{|c|}{ Tipo de hogar } & \multirow[b]{3}{*}{ TOTAL } & \multirow[b]{3}{*}{$\%$} \\
\hline & \multicolumn{2}{|c|}{$\begin{array}{l}\text { Hogares integrados } \\
\text { solamente por } \\
\text { personas adultas } \\
\end{array}$} & \multicolumn{2}{|c|}{$\begin{array}{l}\text { Hogares integrados por } \\
\text { personas adultas y } \\
\text { menores de } 18 \text { años }\end{array}$} & & \\
\hline & Número & $\%$ & Número & $\%$ & & \\
\hline Seguridad & 13 & 5.77 & 95 & 42.22 & 108 & 48.0 \\
\hline Inseguridad leve & 9 & 4.0 & 67 & 29.77 & 76 & 33.77 \\
\hline Inseguridad moderada & 3 & 1.33 & 27 & 12.0 & 30 & 13.33 \\
\hline Inseguridad severa & 1 & 0.44 & 10 & 4.44 & 11 & 4.88 \\
\hline TOTAL & 26 & 11.5 & 199 & 88.4 & 225 & 100 \\
\hline
\end{tabular}

Familias con adultos 
Al analizar las respuestas por pregunta para los tres grupos de alumnos, no se pueden determinar diferencias inseguridad alimentaria, de los cuales uno evidencia inseguridad severa (Cuadro 2), que pertenece a CA (Cuadro 3).

estadísticas. 13 de 26 hogares presentan

Cuadro 3. Distribución de la seguridad alimentaria en hogares de los alumnos del CUCBA integrados solamente por adultos, de acuerdo a la carrera que cursan.

\begin{tabular}{lllll}
\hline \multirow{2}{*}{\begin{tabular}{l} 
Clasificación de la \\
seguridad \\
\cline { 2 - 4 } alimentaria
\end{tabular}} & $\begin{array}{l}\text { Carrera } \\
\text { Ciencia de los }\end{array}$ & $\begin{array}{l}\text { Medicina } \\
\text { Veterinaria }\end{array}$ & Agronegocios & TOTAL \\
\hline $\begin{array}{l}\text { Seguridad } \\
\text { Inseguridad leve }\end{array}$ & 8 & 1 & 4 & 13 \\
$\begin{array}{l}\text { Inseguridad } \\
\text { moderada }\end{array}$ & 3 & 3 & 0 & 9 \\
\begin{tabular}{l} 
Inseguridad severa \\
\multicolumn{1}{c}{ TOTAL }
\end{tabular} & 1 & 0 & 0 & 3 \\
\hline
\end{tabular}

Familias con adultos y menores de 18 años

$\mathrm{Al}$ analizar las respuestas por pregunta para los tres grupos de alumnos, fueron significativas las preguntas "En los últimos 3 meses, por falta de dinero u otros recursos: ¿Alguna vez te preocupó que los alimentos se acabaran en tu hogar?" $(p<0.55)$; ¿Alguna vez en tu hogar dejaron de tener una alimentación saludable? $(p<0,25)$; y ¿Alguna vez tú o algún adulto en tu hogar tuvo una alimentación basada en poca variedad de alimentos? $\quad(\mathrm{p}<0.1) . \quad 104 \quad$ hogares presentan inseguridad alimentaria, de los cuales 10 reflejan inseguridad severa (Cuadro 2), 7 son de CA, 2 de MV y 1 de AN (Cuadro 4). Al analizar las carreras de acuerdo al nivel de seguridad alimentaria (Seguridad/Inseguridad) (Cuadro 4), se encontró diferencia significativa $(\mathrm{p}<0.3)$.

Cuadro 4. Distribución de la seguridad alimentaria en hogares de los alumnos del CUCBA integrados por adultos y menores de 18 años, de acuerdo a la carrera que cursan

\begin{tabular}{lllll}
\hline \multirow{2}{*}{$\begin{array}{l}\text { Clasificación de la } \\
\text { seguridad } \\
\text { alimentaria }\end{array}$} & $\begin{array}{l}\text { Ciencia de los } \\
\text { Alimentos }\end{array}$ & $\begin{array}{l}\text { Medicina } \\
\text { Veterinaria }\end{array}$ & Agronegocios & TOTAL \\
\hline $\begin{array}{l}\text { Seguridad } \\
\text { Inseguridad leve }\end{array}$ & 69 & 7 & 19 & 95 \\
$\begin{array}{l}\text { Inseguridad } \\
\text { moderada }\end{array}$ & 22 & 10 & 12 & 67 \\
\begin{tabular}{l} 
Inseguridad severa \\
\multicolumn{1}{c}{ TOTAL }
\end{tabular} & 143 & 2 & 3 & 27 \\
\multicolumn{1}{r}{} & 75 & 1 & 10 \\
\hline
\end{tabular}

La mayor cantidad de respuestas "sí" para ambos tipos de hogares (9 $\sin$ menores y 76 con menores), fue "¿Alguna vez te preocupó que los alimentos se acabaran en tu hogar?". En tanto que la que presentó menor cantidad de ese mismo tipo de respuestas $(2$ y 25 respectivamente), fue ¿Alguna vez tú o 
algún adulto en tu hogar solo comió una vez al día o dejó de comer durante todo un día?

Para la sección de preguntas específicas a "Hogares integrados por personas adultas y menores de 18 años", la pregunta ¿Alguna vez algún menor de 18 años en tu hogar tuvo una alimentación basada en poca variedad de alimentos? Presento la mayor cantidad de respuestas "sí" (34). Y la pregunta ¿Alguna vez algún menor de 18 años en tu hogar comió menos de lo que debía? Reflejó la menor cantidad de respuestas "sí" (17).

\section{Discusión}

El presente estudio es una aproximación a la situación que prevalece al interior de las familias de los alumnos del Centro Universitario como un aporte para entender la dinámica de la seguridad alimentaria y diseñar estrategias congruentes. Lo anterior es apoyado por los comentarios de los encuestados, quienes externaron opiniones como:

"Es momento para dejar de ser solo un código para la universidad y que comiencen a vernos como seres vivos que se cansan, que se enferman y que en ocasiones pueden fallar".

"Nadie sabe cuando giren las cosas... pues, hoy comemos, mañana no sabemos".

En México, de acuerdo a la Encuesta ENSANUT el $44.5 \%$ de la población, cuenta con seguridad alimentaria, $22.6 \%$ presenta inseguridad alimentaria moderada y severa, en tanto que $32.9 \%$ padece inseguridad alimentaria leve (SS/INSP/INEGI, 2019). Lo anterior, es semejante a los hallazgos del presente estudio (48\% con seguridad alimentaria; $33.77 \%$ con inseguridad leve; $18.21 \%$ con inseguridad moderada y severa) y a lo encontrado por Rosas, Córdova, Villegas, Morales (2017), en un estudio realizado en Boca del Río, Veracruz, en el que $49.4 \%$ de los hogares presenta inseguridad alimentaria y nutricia.

Una investigación realizada en la Universidad del Valle de México (UVM), indicó que el $23 \%$ en una población estudiantil de educación básica, no consume ni una fruta al día, mientras que $29 \%$ no consume verduras y el $10 \%$ no desayuna cotidianamente. Esta tendencia en la alimentación, puede continuar y aumentar en estudiantes de nivel universitario como lo revela el presente estudio (UVM, 2015)

Los resultados del presente estudio son similares al realizado por RamosVázquez y colaboradores (2015), en donde el 37\% de los jóvenes encuestados consumen frutas, verduras y carne solo tres veces a la semana. Datos publicados indicaron que el $50 \%$ de las personas encuestadas tiene una alimentación regular y balanceada, por tanto el otro 50 $\%$ presenta algún grado de inseguridad alimentaria (Mercawise, 2016), lo cual concuerda con los hallazgos en esta investigación.

Según el estudio de Broton and Goldrick-Rab (2018), aproximadamente el $50 \%$ de los universitarios presentan algun grado de inseguridad alimentaria y 
GONZÁLEZ-AGUILAR ET AL.

el $20 \%$ (Uno de cada cinco) tienen muy bajo nivel de seguridad alimentaria, lo cual refleja que estan preocupados por su alimentación, ya que es insuficiente. Esto ultimo coincide con los comentarios de los estudiantes encuestados:

"Durante la pandemia se han visto afectadas familias como la mía, que consumían alimentos al día".

"Si bien nunca se dejo de comer, no hubo una alimentación balanceada por falta de dinero".

"Sí es necesario tener dinero para alimentarse variada y sanamente".

"En mi familia hemos pasado por limitaciones de comida por falta de dinero, este confinamiento a muchas personas nos afecto en el trabajo, se trabajan 48 horas a la semana, mas las horas de estudio y disminuyeron los sueldos, otros perdieron su empleo y es hora que no se pueden acomodar económicamente".

Broton and Goldrick-Rab (2018), señalan al respecto: los estudiantes que viven con inseguridad alimentaria, no pueden permitirse el acceso a una adecuada alimentación (ingerir comidas balanceadas), aunque hagan el esfuerzo, ya que no pueden comprar más por falta de dinero, incluso el 36\% señalan tener hambre y no poder comer por falta de dinero para alimentarse. Hasta el 11\% manifiestan que han pasado un día sin comer por ausencia de dinero.

En un estudio basado en el censo de los E.U. 2001-2015, encontraron que de 2008 a 2014, 21.2 \% de los hogares incluyen estudiantes en universidades de dos años, que reportan experiencia de inseguridad alimentaria. Cerca del $13 \%$ de los estudiantes de universidades de cuatro años presentaron la misma condición durante ese período. En 2015 los niveles de inseguridad alimentaria fueron de $11.2 \%$ en estudiantes de universidades de cuatro años, lo cual es similar a los niveles nacionales en E.U (Blagg, Gundersen, Whitmore and Ziliak. 2017). Dichos datos contrastan con la realidad Mexicana ya mencionada en la encuesta ENSANUT (SS/INSP/INEGI, 2019).

$20 \%$ de los estudiantes en la Universidad de California (UC), viven en "Inseguridad alimentaria". Los cuales reportan niveles excesivos de estres y refieren que éste puede ser extremadamente distractor en clases ya que tienen que manejar los requerimientos escolares y su vida personal (California State University [CSU], 2015). Estos efectos del estrés, es muy probable que se presenten con mayor frecuencia en los estudiantes del CUCBA.

En otro estudio realizado por la UC, un $19 \%$ de los estudiantes sufrían de una seguridad alimentaria muy baja, es decir, experimentaron un consumo reducido de alimentos en ocasiones debido a tener recursos limitados. Un $23 \%$ tuvieron una inseguridad alimentaria "baja" (Una dieta de calidad, variedad y deseabilidad reducidas). En total en dicho estudio, un $42 \%$ de los estudiantes mostraron inseguridad alimentaria (Martinez, Maynard y Ritchie, 2016). Cifra muy cercana a lo reflejado en el estudio que nos ocupa.

En estudios posteriores es necesario considerar las características de los 
hogares de los estudiantes, ya que es un factor importante para la seguridad alimentaria. Broton y Goldrick-Rab (2018), señalan que aproximadamente el $33 \%$ de los estudiantes tienen viviendas inseguras, de los cuales $14 \%$ no tienen hogar, lo cual influye en el nivel de inseguridad alimentaria.

\section{Posibles estrategias de intervención}

Los estudiantes que participaron en el estudio hicieron aportaciones a este respecto, que coinciden en lo general con estrategias documentadas en la literatura pero que deben ser analizadas profundamente.

Algunas instituciones educativas en Estados Unidos se han incorporado al programa "Swipe Out Hunger" (Eliminar el hambre), que se basa en un programa anterior con el uso de cupones del plan de comidas, los estudiantes que no los utilizan los donan a otros estudiantes para ser usados en los comedores del campus o en los bancos de alimentos, también les proporcionan despensas (Martinez, Maynard y Ritchie, 2016; Universidad de California, 2017).

La UC, creó el Paquete de Herramientas para el Acceso y Seguridad Alimentaria de los Estudiantes (Student Food Access and Security Toolkit). El paquete compila una serie de mejores prácticas que se han desarrollado en los campus de UC aunado a la promoción para nutrir y apoyar a los estudiantes (Martinez, Maynard y Ritchie, 2016; Universidad de California, 2017).

\section{Conclusión}

Los datos presentados sugieren una realidad preocupante en torno a la seguridad alimentaria en las familias de los estudiantes del Centro Universitario, cinco de cada 10 estudiantes viven en familias con inseguridad alimentaria, es decir, los estudiantes están sufriendo hambre y malnutrición y se muestran preocupados por ello.

Se requiere de investigaciones complementarias que ahonden sobre la correlación de los hogares (incluida la vivienda misma) y el número de miembros de la familia que aportan dinero para poder comer adecuadamente, con la inseguridad alimentaria y sus causas con la eficiencia del aprendizaje de los alumnos.

Las estrategias deben ser consideradas para el diseño de políticas institucionales de apoyo a los estudiantes en condición de vulnerabilidad, que repercutan en profesionistas mejor preparados, sin menoscabo de otras esferas que se tengan que tomar en cuenta. Un enfoque específico es mejor para resolver problemas de inseguridad alimentaria en la educación superior, orientado a un mejor rendimiento académico. 


\section{Literatura citada}

Altieri, M., P. Koohafkan y E. H. Giménez. (2012). "Agricultura verde: fundamentos agroecológicos para diseñar sistemas agrícolas biodiversos, resilientes y productivos". Agroecología, 7(1), 7-18. https://revistas.um.es/agroecologia/artic le/view/170961/146181

Blagg, K., C. Gundersen, S.D.Whitmore y J.P. Ziliak. 2017. Assessing Food Insecurity on Campus, A National Look at Food Insecurity among America's College Students. Education Policy Program. Urban Intitute. [Publicación en línea], disponible desde internet en

$<$ https://www.urban.org/sites/default/files/ publication/92331/assessing_food_inse curity_on_campus_4.pdf $>$. [Con acceso el 06-febrero-2021].

Broton, K.M. and S. Goldrick-Rab. (2018). Going Without: An Exploration of Food and Housing Insecurity Among Undergraduates. Educational Researcher, 47( 2), 121-133. https://doi.org/10.3102/0013189X1774 1303

California State University [CSU]. 2015. Serving Displaced and Food Insecure Students in the CSU. [Publicación en línea], disponible desde internet en

$<$ https://www2.calstate.edu/impact-of-the$\mathrm{csu} /$ student-success/basic-needs initiative/Documents/ServingDisplaced andFoodInsecure

StudetnsintheCSUJanuary20163.8.16.p df $>$. [Con acceso el 04-febrero-2021].

Coleman-Jensen, A., M. Rabbitt, C. Gregory, and A. Singh. 2016. Household Food Security in the United States in 2015. Economic Research Report No. (ERR-215). Washington, DC: US Department of Agriculture. [Publicación en línea], disponible desde internet en
$<$ https://www.ers.usda.gov/webdocs/publi cations/79761/err-215.pdf $>$ [Con acceso el 28-enero-2021].

Consejo Nacional de Evaluación de la Política de Desarrollo Social [CONEVAL]. 2019. Diez años de medición de pobreza multidimensional en México: avances y desafíos en política social. Medición de la pobreza serie 2008-2018. [Publicación en línea], disponible desde internet en $<$ https://www.coneval.org.mx/Medicio $\mathrm{n} /$ Paginas/PobrezaInicio.asp $>$. [Con acceso el 18-abril-2020].

Escalona-Aguilar, M.A., M.T. LealAscencio, M.R. Pineda-López, E.E. Ruíz-Cervantes, L.R.SánchezVelásquez. (2015). El Papel De La Universidad Pública en la Soberanía Alimentaria. Revista Mexicana de Investigación Educativa RMIE, 20(67), 1215-1231.

$<$ http://www.scielo.org.mx/pdf/rmie/v2 0n67/v20n67a10.pdf $>$.

Goldrick-Rab, S., K. Broton, and D. Eisenberg. (2015). Hungry to Learn: Addressing Food and Housing Insecurity among Undergraduates. Madison, WI: Wisconsin Hope Lab. [Publicación en línea], disponible desde internet en <https:// hope4college.com/wpcontent/uploads/2018/09/Wisconsin HOPE_Lab_Hungry_To_Learn.p $\bar{d} f>$. [Con acceso el 19-enero-2021]

Gundersen, C., and J. P. Ziliak. (2015). "Food Insecurity and Health Outcomes." Health Affairs, 34(11), 1830-39.

https://www.healthaffairs.org/doi/pdf/1 0.1377/hlthaff.2015.0645

Knol, L.L., C.A. Robb, E.M. McKinley, and M.Wood. (2017). "Food Insecurity, Self-Rated Health, and Obesity among College Students." American Journal of 
Health Education, 48(4), 248-55. https://doi.org/10.1080/19325037.2017. 1316689

Lachat, C., E. Nago, R. Verstraeten, D. Roberfroid, J. Van Camp, y P. Kolsteren. (2012). Eating out of home and its association with dietary intake: a systematic review of the evidence. Obesity Reviews, 13 (4), 329-346. doi: $10.1111 / \mathrm{j} .1467-$ 789X.2011.00953.X

López, I., A. Sánchez, I. Johansson, J. Petkeviciene, R. Prattala and M. Martínez. (2003). Disparities in food habits in Europe: systematic review of educational and occupational differences in the intake of fat. Journal of Human Nutrition and Dietetics, 16(5), 349-364. DOI: 10.1046/j.1365277x.2003.00466.x

Martinez, S.M., K. Maynard and L.D. Ritchie. Student Food Access and Security Study. 2016. University of California. Global Food Initiative. [Publicación en línea], disponible desde internet

$<$ https://www.ucop.edu/global-foodinitiative/best-practices/food-accesssecurity/student-food-access-andsecurity-study.pdf $>$. [Con acceso el 09febrero-2021].

Mercawise. Encuesta de Mercado. 2016. Hábitos alimenticios en México. [Publicación en línea], disponible desde internet en $<$ https://www.mercawise.com/blog/estu dios-de-mercado/habitos-alimenticiosen-mexico/>. [Con acceso el 03febrero-2021].

Organización de las Naciones Unidad para la Alimentación y la Agricultura [FAO]. 2012. Escala Latinoamericana y Caribeña de Seguridad Alimentaria (ELCSA). [Publicación en línea], disponible desde internet en $<$ http://www.fao.org/3/a-i3065s.pdf $>$.

[Con acceso el 19-marzo-2020].
Organización de las Naciones Unidad para la Alimentación y la Agricultura [FAO]. 2019a. Día Mundial de la Alimentación. Nuestras acciones son nuestro futuro. Una alimentación sana para un mundo \#hambrecero. [Publicación en línea], disponible desde $<$ http://www.fao.org/world-foodday/theme/es/ $>$. [Con acceso el 10noviembre-2020].

Organización de las Naciones Unidad para la Alimentación y la Agricultura [FAO]. 2019b. Día Mundial de la Alimentación. Receta para una vida saludable. Todo el mundo puede desempeñar su papel. [Publicación en línea], disponible desde $<$ http://www.fao.org/world-foodday/take-action/es/>. [Con acceso el 10-noviembre-2020].

Organización de las Naciones Unidad para la Alimentación y la Agricultura/Fondo Internacional de Desarrollo Agrícola/Organización Panamericana de la Salud/Programa Mundial de Alimentos/Fondo de las Naciones Unidas para la Infancia [FAO/FIDA/OPS/WFP /UNICEF]. 2020. Panorama de la seguridad alimentaria y nutricional en América Latina y el Caribe 2020. Seguridad alimentaria y nutricional para los territorios más rezagados. Santiago de Chile. [Publicación en línea], disponible desde internet en $<$ https://doi.org/10.4060/cb2242es $>$.

[Con acceso el 01-febrero-2021].

Ramos-Vázquez, Salazar-Peña, GarcíaMadrid, Hernández-Gutiérrez, BonillaLuis, Pérez-Noriega. (2015) Hábitos de Alimentación en Estudiantes Universitarios. XII Encuentro "Participación de la Mujer en la Ciencia". [Publicación en línea], disponible desde internet en $<$ http://congresos.cio.mx/memorias_co ngreso_mujer/archivos/Memorias_201 
5.pdf $>$. [Con acceso el 10-febrero2020].

Rosas, S.T.J., O.A.P. Córdova, A.J.G. Villegas, B.N. Morales. (2017). Evaluación de la Inseguridad Alimentaria y Nutricia de Escolares y sus Familias. Revista Médica de la Universidad Veracruzana, 17(1), 7-22. https://www.medigraphic.com/cgibin/new/resumen.cgi?IDARTICULO= 73355

Secretaría de Salud /Instituto Nacional de Salud Pública/Instituto Nacional de Información Estadística y Geográfica [SS/INSP/INEGI]. 2019. Encuesta Nacional de Salud y Nutrición 2018. [Publicación en línea], disponible desde internet en $<$ https://ensanut.insp.mx/encuestas/ens anut2018/doctos/informes/ensanut_201 8 presentacion_resultados.pdf $>$. [Con acceso el 13-abril-2021].

Statgraphics 18 - X64, 2019. Version 18.1.12 posted July 10, 2019. Statgraphics Technologies, Inc.

Universidad del Valle de México [UVM]. 18 Nov. 2015. 91\% de los niños y jóvenes en México consume más de 5 porciones de bebidas azucaradas al día. [Publicación en línea], disponible desde internet en $<$ https://aureatecomunicacion.com/prensa/91-de-losninos-y-jovenes-en-mexico-consumemas-de-5-porciones-de-bebidasazucaradas-al-dia/\#.YBxEYDEzbak>. [Con acceso el 09-febrero-2021].

University of California. Global Food Initiative: Food and Housing Security at the University of California. Executive Summary. December 2017. [Publicación en línea], disponible desde internet en $<$ https:/www.ucop.edu/global-foodinitiative/_files/food-housing-securityexec-summary.pdf $>$. [Con acceso el 27-enero-2021] 